\title{
High Prevalence of Extended-Spectrum Beta- Lactamase-Producing Respiratory Bacterial Pathogens in a Nepalese University Hospital: A Vexatious Problem
}

\author{
Shyam Kumar Mishra ${ }^{1 *}$,Hari Kattel ${ }^{1}$, Bharat Mani Pokhrel', Basista Prasad Rijal ${ }^{1}$
}

BACKGROUND: Extended-spectrum beta-lactamase (ESBL)-producing bacteria have resulted in vexatious problem to combat lower respiratory tract infection (LRTI). However, no detailed studies have been done in Nepal exploring the status of such pathogens from LRTI. Therefore, this study was carried out to assess the current levels of antimicrobial resistance with special reference to ESBL-producing respiratory bacterial pathogens in Nepal.

METHODS: A prospective study was conducted at Tribhuvan University Teaching Hospital in the capital city of Nepal. Specimens representing lower respiratory tract were received from 1120 patients suspected of LRTI. The specimens were collected and processed according to standard methodology. Combination disk (CD) and disk synergy test (DST) methods were used for the detection of ESBLproducing isolates.

RESULTS: Of the total 314 isolates belonging to Enterobacteriaceae and nonfermentative bacteria, 24.2\% were ESBL-producers which included $42.2 \%$ of Klebsiella pneumoniae, $8.8 \%$ of Pseudomonas species, $41.9 \%$ of Escherichia coli, $12.9 \%$ of Acinetobacter species, $13.3 \%$ of Citrobacter species and $66.7 \%$ of Morganella morganii. ESBL-producers were more common among hospitalized patients. For ESBL-producers, the most effective drug was found to be imipenem, meropenem, followed by cefoperazone-sulbactam and amikacin.

CONCLUSIONS: One-fourth of the isolates belonging to Enterobacteriaceae and non-fermentative bacteria were ESBL-producer. It is quite essential to have effective antimicrobial and infection control policy in the hospital and special steps should be taken to prevent clonal dissemination of the resistant strains. Further studies should be done in other hospitals of Nepal to know the phenotype and genotype of ESBL-producing isolates prevailing in our country.

KEY WORDS: Antimicrobial resistance, ESBL, Respiratory pathogens

(C) 2015 Nepalese Association for Clinical Chemistry

\section{Introduction}

Acute Lower Respiratory Tract Infection (LRTI) is one of the principal causes of the morbidity and mortality in the world [1]. Almost threequarters of all antibiotic consumption are for respiratory tract infections [2]. Beta ( $\beta$ )-lactams remain a cornerstone for antimicrobial chemotherapy of a large number of bacterial infections [3] including LRTI, but their efficacy has been increasingly thwarted by dissemination of acquired resistance determinants among pathogenic bacteria.

The exposure of bacterial strains to a large number of $\beta$-lactams has induced a dynamic production and mutation of $\beta$-lactamase in many bacteria, escalating their activity even against the later generation cephalosporins [4] by the production of extended-spectrum $\beta$-lactamase (ESBL) and/or AmpC $\beta$-lactamase. These enzymes are often associated with multidrug resistance. AmpC- $\beta$-lactamases, in contrast to ESBLs, hydrolyze broad and extended-spectrum cephalosporins (cephamycins as well as to oxyimino- $\beta$-lactams) but are not inhibited by $\beta$ lactamase inhibitors such as clavulanic acid. Although ESBL- and AmpC-producing strains are typically susceptible to carbapenems, diminished porin expression can make such strains carbapenem-resistant as well.

The resistance mechanisms like ESBL and AmpC are already disseminated on a worldwide scale. The first prevalence study of ESBLproducing bacterial isolates in Nepal showed that more than $20 \%$ of clinical isolates were positive for ESBL [5]. However, earlier studies cannot precisely reflect the current status of novel $\beta$ lactamases. Despite newer guidelines by European Committee on Antimicrobial Susceptibility Testing (EUCAST) and Clinical

\footnotetext{
'Department of Microbiology, Institute of Medicine, Tribhuvan University Teaching Hospital, Kathmandu, Nepal
} 
and Laboratory Standards Institute (CLSI), researchers have recommended continuing to seek ESBLs directly and, where they are found, generally to avoid substrate drugs as therapy [6].Therefore, the determination of current status of ESBL-producing bacteria following standard methodology is crucial in our context for which this study was conducted among the patients attending Tribhuvan University Teaching Hospital (TUTH), Kathmandu.

\section{Methods}

This was a cross-sectional study conducted over a period of six months in the bacteriology laboratory of TUTH. A total of 1120 lower respiratory tract representing specimens received for culture and sensitivity were processed following standard microbiological method [7]. The specimens included sputum $(\mathrm{n}=1039)$, endotracheal (ET) secretion $(\mathrm{n}=61)$ and bronchial washing $(\mathrm{n}=20)$.

Identification of significant isolates was done following standard microbiological techniques which involved morphological appearance of the colonies, Gram's staining reactions, catalase test, oxidase test with other biochemical properties [7]. Firstly, pure form of the culture was obtained from the primary culture by using purity plate and then it was processed for biochemical tests. The biochemical media employed were - Triple Sugar Iron (TSI) agar, Sulphide Indole Motility (SIM) media, Simmon's citrate media, Christensen's urea agar, Decarboxylase test media, Hugh and Leifson's Oxidation-Fermentation test media, Glucose phosphate broth (Methyl red/VogesProskauer test), Phenylalanine agar (Oxoid, UK) and others as required.

The antibiotic sensitivity testing of the bacterial pathogens was performed on Mueller Hinton agar (MHA) (Oxoid, United Kingdom) by Kirby-Bauer disk diffusion method as recommended by CLSI [8]. For disk susceptibility testing, ciprofloxacin, cotrimoxazole, gentamicin, amikacin, ceftazidime, ceftriaxone, cefotaxime, cefepime, piperacillin, piperacillin-tazobactam, cefoperazone-sulbactam, meropenem and imipenem were used. Escherichia coli American Type Culture Collection (ATCC) 25922 and Pseudomonas aeruginosa ATCC 27853 strains were also tested, in every set of experiment, in parallel, as a part of quality control. In this study, if the isolates were resistant to at least three classes of first line antimicrobial agents, they were regarded as multidrug resistant (MDR) [9].

Tests for ESBL-production in Gram-negative isolates

\section{SCREENING TEST}

The initial screening for the production of ESBL was performed by using ceftriaxone $(30 \mu \mathrm{\mu})$, ceftazidime $(30 \mu \mathrm{g})$ and cefotaxime $(30 \mu \mathrm{g})$ disks (Oxoid, United Kingdom). If the zone of inhibition (ZOI) was $\leq 25 \mathrm{~mm}$ for ceftriaxone, $\leq$ $22 \mathrm{~mm}$ for ceftazidime and/or $\leq 27 \mathrm{~mm}$ for cefotaxime, the isolate was considered a potential ESBL-producer as recommended by CLSI [8]. The organism was swabbed on to a MHA plate as done for screening test in antibiotic sensitivity test. Then combination disk (CD) and disk synergy test (DST) methods were applied.

\section{METHOD}

$\mathrm{CD}$ method was also used for the phenotypic confirmation of ESBL-producing strains in which cefotaxime and ceftazidime $(30 \mu \mathrm{g})$, alone

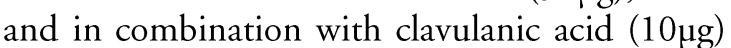
were used (Becton Dickinson, USA). An increased ZOI of $\geq 5 \mathrm{~mm}$ for either antimicrobial agent tested in combination with clavulanic acid versus its zone when tested alone confirmed ESBL [8].

\section{DST}

Isolates suspected as ESBL-producer by screening test were tested further by DST [10]. In this method, amoxycillin-clavulanic acid disk $(20 / 10 \mu \mathrm{g})$ was placed at the center and disks

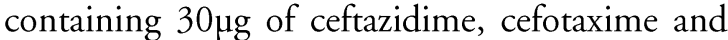
ceftriaxone were placed separately beside $15 \mathrm{~mm}$ distance (edge to edge), away from the central disk [11, 12]. Any enhancement of the ZOI between the disks (any of the cephalosporin disks and clavulanic acid containing disk) indicated the presence of ESBL [13]. E. coli ATCC 25922 and $K$. pneumoniae ATCC 700603 were used as negative and positive controls respectively.

In this study, if repeat cultures were received at intervals of less than every 48 hour, the specimens were rejected. Besides, specimens not fulfilling the criteria of American Society for Microbiology were rejected [7]. Sputum and ET secretions were processed further only if they had more than 25 leucocytes and fewer than 10 epithelial cells per low power field of microscope. Ethical approval was taken from Institutional Review Board of Institute of Medicine to conduct this study. Data were analyzed using Microsoft Excel and interpreted according to 
frequency distribution, percentage. Chi-square test was used to determine significant association of different variables and $P$ value of $<0.05$ was regarded as significant.

\section{Results}

Among the total 533 pathogenic bacterial isolates, 314 belonged to Enterobacteriaceae and non-fermentative bacteria. Among them, 76 (24.2\%) were ESBL-producer and $25(8.0 \%)$ were screening test positive for AmpC $\beta$ lactamase.

ESBL-production was seen in Klebsiella pneumoniae, Pseudomonas spp., Acinetobacter spp., Escherichia coli, Citrobacter spp. and Morganella morganii. Of the total 102 isolates of $K$. pneumoniae, 43 were ESBL-producer. Around $12.9 \%$ of Acinetobacter isolates were ESBL-producer. Among the $37 \mathrm{E}$. coli isolates, $13(35.1 \%)$ were positive for ESBL. Out of three $M$. morganii isolates, two were ESBL-producing (Figure 1).

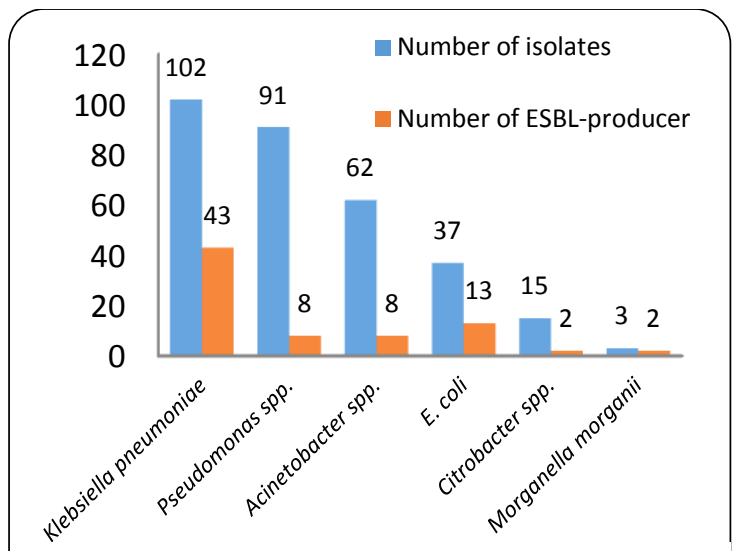

Figure 1. ESBL-producing gram-negative bacteria

ESBL-producers were significantly higher among the inpatients $(\mathrm{n}=59 ; 77.6 \%)$ as compared to outpatients $(\mathrm{n}=17 ; 22.4 \%)$. Isolates from medical wards $(\mathrm{n}=24)$ and intensive care unit (ICU) $(\mathrm{n}=23)$ were found to carry relatively higher frequency of ESBL followed by cardiac care unit $(n=6)$ and surgical unit $(n=6)$.
This study revealed that $71(93.4 \%)$ of the ESBL-producing isolates were sensitive to different antibiotics as follows- imipenem $(\mathrm{n}=71$; 93.4\%), meropenem $(\mathrm{n}=61 ; \quad 80.3 \%)$, cefoperazone-sulbactam $\quad(\mathrm{n}=54 ; \quad 71.1 \%)$, amikacin $(\mathrm{n}=52 ; 68.4 \%)$ and piperacillintazobactam $(\mathrm{n}=48 ; 63.2 \%)$ (Table 1$)$.

Among the 221 Enterobacteriaceae and nonfermentative isolates subjected to two different phenotypic ESBL detection methods, combination disk method detected 76 (34.4\%) and DST method detected 63 (28.5\%) cases.

Of the total ESBL positive isolates by DST method, the following numbers of isolates gave clavulanate enhanced synergistic zones with the $\beta$-lactam disk - ceftriaxone ( $\mathrm{n}=61 ; 96.8 \%)$, cefotaxime $(\mathrm{n}=60 ; 95.2 \%)$ and ceftazidime $(\mathrm{n}=63 ; 100.0 \%)$ (Figure 2).

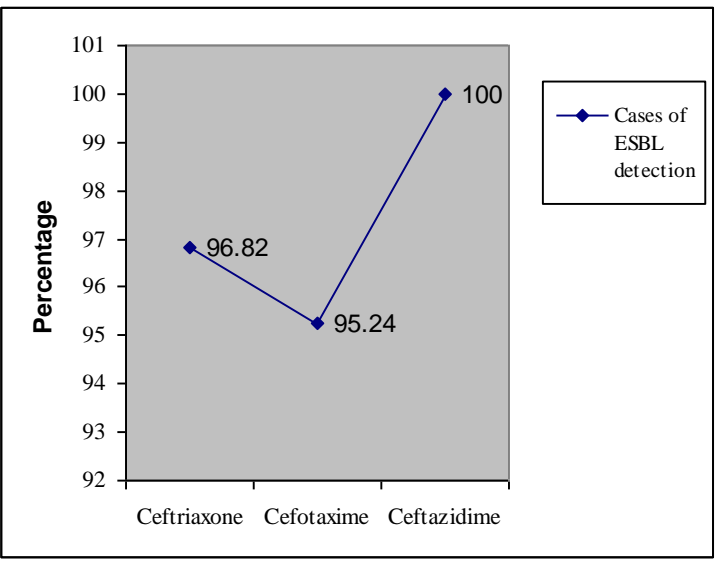

Figure 2. Ceftriaxone, cefotaxime and ceftazidime sensitivity in DST method

It was interesting to note that out of 107,100 and 122 isolates that were susceptible to ceftriaxone, cefotaxime and ceftazidime respectively, $8.4 \%(9 / 107), 7 \%(7 / 100)$ and $12.3 \%(15 / 122)$ were found to be ESBLproducers.

\section{Discussion}

Incidence of antibiotic resistance has increased for respiratory pathogens in such a way that it

Table 1. Antibiogram of ESBL-producing bacterial isolates $(\mathbf{n}=76)$

\begin{tabular}{|c|c|c|c|c|}
\hline \multirow{2}{*}{ Antibiotics } & \multicolumn{2}{|c|}{ Sensitive } & \multicolumn{2}{|c|}{ Resistant } \\
\hline & No. & $\%$ & No. & $\%$ \\
\hline Ciprofloxacin & 15 & 19.7 & 61 & 80.3 \\
\hline Cotrimoxazole & 7 & 9.2 & 69 & 90.8 \\
\hline Gentamicin & 31 & 40.8 & 45 & 59.2 \\
\hline Amikacin & 52 & 68.4 & 24 & 31.6 \\
\hline Piperacillin-Tazobactam & 48 & 63.2 & 28 & 36.8 \\
\hline Imipenem & 71 & 93.4 & 5 & 6.6 \\
\hline
\end{tabular}


has complicated the use of empiric treatment with traditional agents [14]. It is interesting to note that bacteria are capable of phenotypic switching of antibiotic resistance which may further result in confusion in treatment [15]. Therefore, antibiogram of the clinical isolates must be generated and the patients should be treated accordingly.

In this study, the decreased susceptibility of gram-negative isolates to third and fourth generation cephalosporins: cefotaxime, ceftriaxone, ceftazidime and cefepime $(26.8 \%$ to $28.6 \%$ ) could be attributed to ESBL- or AmpC $\beta$-lactamase-production or some other relevant underlying mechanisms. This study showed $24.2 \%$ of the gram-negative isolates were ESBLproducers (this accounts for $14.3 \%$ of the total bacterial isolates). ESBL production was most common among $K$. pneumoniae $(42.2 \%)$ which was followed by E. coli (41.9\%), Citrobacter spp. $\quad(13.3 \%)$, Acinetobacter calcoaceticus baumannii complex (12.9\%), Pseudomonas spp. $(8.8 \%)$ and $M$. morganii. Previous study in 2004 at the same hospital had shown $24.27 \%$ of the total isolates $(40.39 \%$ of the gram-negative isolates) were positive for ESBL. In that study, $55.0 \%$ of K. pneumoniae, $50.0 \%$ of E. coli, and $20.69 \%$ of Pseudomonas spp. were ESBLproducer [16]. A relatively lower prevalence of ESBL-producing isolates was seen in another study in Nepal $(28.6 \%$ of E. coli, $8.3 \%$ of $K$. pneumoniae and $2.4 \%$ of $P$. aeruginosa) [17]. However, genotypic confirmation of the ESBLpositive nonfermenters in further studies is needed as phenotypic tests can give false-positive results. Moreover, AmpC production in Citrobacter spp. and M. morganii can mask additional ESBL-production; therefore, a genotypic confirmation of all Citrobacter spp. and M. morganii is needed.

The prevalence of ESBL among clinical isolates varies from country to country and institution to institution [18]. This may be ascribed to local antibiotic prescribing habits and presence of pathogens harboring the gene for ESBL production. It has been noted that ESBLproducing isolates are increasing globally [19]. Fortunately, this study showed a declination in the figure of ESBL producers among LRTI isolates at TUTH when compared with previous study [16]. However, the trend should further be monitored closely in days to come.

The SENTRY Antimicrobial Surveillance Programme (1998-2004) among LRTI pathogens in Europe reported that ESBL phenotypes remained essentially unchanged among E. coli between 1998 and 2004 (8.2\% and $8.3 \%$, respectively), whereas among $K$. pneumoniae, increases were more substantial (16.7\% and 26.9\%) [20]. The high incidence of Klebsiella spp. might be due to their longer survival than other enteric bacteria on hands and environmental surfaces, facilitating crossinfection within hospitals [21]. Though ESBLproducing $K$. pneumoniae were found in less number than previous study in our setting, this study depicts the increasing trend of ceftazidime resistance which was $28.9 \%$ in $2006,60.4 \%$ in 2007 and $69.6 \%$ in 2008 [22]. This could be attributed to other mechanisms of third generation cephalosporins resistance (besides ESBL production), like due to AmpC $\beta$ lactamase production.

In this study, ESBL-producers were more prevalent among members of Enterobacteriaceae and all the ESBL-producing isolates were MDR. ESBL production is coded by genes that are prevalently located on large conjugative plasmids of $80-160 \mathrm{~kb}$ in size [23]. Since these plasmids are easily transmitted among different members of the Enterobacteriaceae, accumulation of resistance genes results in strains that contain multiresistant plasmids. Therefore, ESBLproducing isolates may confer resistance to different classes of antibiotics as shown in other studies [16-24].

For ESBL-producers, the most effective drug was found to be imipenem, followed by meropenem, cefoperazone-sulbactam, amikacin and piperacillin-tazobactam. However, there was no statistically significant difference between their sensitivity figure against ESBL- producers and non-producers (data not shown). When these antimicrobials were compared regarding their efficacy against ESBL-producers, the difference of piperacillin-tazobactam and cefoperazonesulbactam was not considerable $(P>0.05)$; nevertheless, imipenem showed significant efficacy as compared to aforementioned combination drugs $(P<0.001)$. However, always jumping directly on carbapenems for cephalosporin resistant bacteria may welcome carbapenem resistance as seen in this study. In India, $22.2 \%$ and $17.3 \%$ of ESBL-producers showed resistance to meropenem and imipenem respectively [19]. We found carbapenem resistance in isolates of ESBL-producing $A c b$ complex, Pseudomonas spp., Klebsiella pneumoniae and Citrobacter spp. The resistance of several isolates to imipenem and meropenem 
in this study raised the suspicion that the ESBLpositive isolates were co-producer of carbapenemase(s).

$\beta$-lactam and $\beta$-lactamase inhibitor combinations are usually active against organisms possessing a single ESBL. However, many organisms produce multiple ESBLs which may reduce the efficacyof $\beta$-lactam/ $\beta$-lactamase inhibitor combinations. In vitro resistance of ESBL-producing isolates to such combinations is increasing [25]. The present study showed relatively higher susceptibility of ESBL-producers towards piperacillin-tazobactam which indicates that PER-1 is relatively uncommon in Nepal as compared to other ESBL types. However, the genotyping characterization of ESBL was not done in this study. It should be noted that PER-1 is resistant to tazobactam [25].

The effectiveness of gentamicin was found better for non-ESBL-producers than for ESBLproducers $(65.55 \%$ vs. $40.79 \%, P<0.001)$. As ESBL genes occur predominantly on large plasmid carrying multiple resistance, this might have resulted in higher prevalence of gentamicin resistance among ESBL-producing isolates. Likewise, higher resistance was observed among ESBL-producing isolates as compared to nonESBL producers against other antibiotics testedciprofloxacin $(19.74 \%$ vs. $57.98 \%, P<0.001)$, cotrimoxazole $(9.21 \%$ vs. $27.73 \%, P<0.001)$, piperacillin $(5.26 \%$ vs. $60.50 \%, \quad P<0.001)$, ceftazidime $(3.95 \%$ vs. $36.97 \%, P<0.001)$ and cefepime $(5.26 \%$ vs. $38.66 \%, P<0.001)$.

There have been increasing reports of plasmidencoded resistance to quinolones, frequently in association with plasmid-mediated cephalosporin resistance [21]. There is a strong association between quinolone resistance and ESBL production even when plasmid-encoded decreased quinolone susceptibility is not present. The reason for this association is not well understood [21]. Gunseren et al [25] found that ESBL producers showed $41.3 \%$ and $48.2 \%$ sensitivity to amikacin and ciprofloxacin respectively while they showed $92.0 \%$ sensitivity with imipenem, comparable to the present study.

Out of total 76 ESBL-producing isolates, $77.6 \%$ belonged to inpatients while remaining $22.4 \%$ were from outpatients. This suggests that there is a threat of drug resistance and dissemination of such strains not only in hospitals but also in the community. Among inpatients, a higher number of the isolates were from medical wards and ICUs. In Turkey, ESBL-producers among ICU isolates were found to range from $21.1 \%$ to
$58.0 \%$ in different hospitals [26]. Third generation cephalosporin such as ceftriaxone, cefotaxime and ceftazidime are extremely used in ICUs and other medical settings even in our part. Therefore, the resistance observed here may have appeared under the selective influence of the extensive usage of these antibiotics. Moreover, the specific risk factors applicable to ICU patients include length of hospital stay, severity of illness, duration of stay in the ICU, and mechanical ventilation.

The ICU isolates harboring ESBL showed resistance rate of more than $60 \%$ against cephalosporins, aminoglycosides and quinolones. Though imipenem stood out as the agent with the lowest resistance rate, the resistance rate was still higher (more than 40\%). This rate is higher than report in India by Gupta et al $37.3 \%$ isolates resistant to meropenem, $31.9 \%$ to imipenem) [19]. Likewise, cefoperazonesulbactam and meropenem were found to have mediocre activity with sensitivity rate of $40.0 \%$ to $43.0 \%$. However, in a study [27], low resistance level in $P$. aeruginosa against piperacillin (4-14\%), piperacillin-tazobactam (3$8 \%$ ), ceftazidime (0-4\%), gentamicin (2-9\%), and ciprofloxacin (7-11\%) was noted in ICU isolates.

Interestingly, ESBL-producing organisms may appear susceptible to some extended-spectrum cephalosporins. However, treatment with such antibiotics has been associatedwith high failure rates [21]. It is well known that poor outcomes occur when patients with serious infections due to ESBL-producers are treated with antibiotics to which the organisms are resistant [26]. In this study, $8.0 \%$ to $12.0 \%$ of the gram-negative isolates, susceptible to ceftriaxone, cefotaxime or ceftazidime, were found to be ESBL-producer. Therefore, accurate detection and reporting of ESBL production in clinical isolates are crucial [28]. For this purpose, total 221 primary screening test positive isolates were subjected to DST and CD method for the phenotypic confirmation; the latter method detected more ESBL cases $(P>0.05)$ which was in agreement to the result shown in other studies $[11,29]$. The CD method detected thirteen more cases than DST method. A major advantage of the DST method is that it is technically simple; however, the interpretation of the test is quite subjective. Sensitivity may be reduced when ESBL activity is very low, leading to wide zones of inhibition around the cephalosporin disks. This has been noted while testing ESBL detection in Proteus 
mirabilis [21].

In this study, three different third generation cephalosporin disks were used for screening ESBL. Ceftazidime detected all the ESBLproducers, while cefotaxime and ceftriaxone could not. In a study, $89 \%$ of $K$. pneumoniae isolates met the criteria for a positive phenotypic confirmatory test with both ceftazidime and cefotaxime, $9 \%$ with ceftazidime only and $2 \%$ with cefotaxime only [30]. The use of ceftazidime alone results in the inability to detect CTX-M-producing organisms [21]. It is emphasized that different cephalosporins with and without clavulanate should be used for the test.

\section{Conclusions}

There is still a great threat to patient management due to ESBL-producing isolates. To overcome this problem, there should be a vigilant detection of such isolates in the laboratory. The high prevalence of ESBL has strictly indicated the need for special hospital care for the management of such patients and the prevention of dissemination of such strains. Last but not the least, genotypic characterization of all ESBL-phenotypes should be done in further studies to compare the results with other countries and to see whether there is clonal spread of such strains in our setting.

Conflict of Interest: None declared

\section{REFERENCES}

1. Murray CJL, Lopez AD. Mortality by cause for eight regions of the world: Global Burden of Disease Study. Lancet. 1997; 349:126976.http://dx.doi.org/10.1016/S0140 -6736(96)07493-4

2. File TM. The epidemiology of respiratory tract infections. Semin Respir Infect. 2000; 15:18494.http://dx.doi.org/10.1053/srin.2 000.18059

3. Cornaglia G, Giamarellou H, Rossolini GM. Metallo-betalactamases: a last frontier for betalactams? Lancet Infect Dis. 2011; 11(5):381-

93.http://dx.doi.org/10.1016/S1473 -3099(11)70056-1

4. Araj GF, Samaha-Kfoury JN. Recent developments in betalactamases and extended spectrum beta lactamases. BMJ. 2003; 327:1209-1213.

http://dx.doi.org/10.1136/bmj.327. 7425.1209

5. Mishra SK, Koirala J, Pokhrel BM, Dahal RK, Khadga PK, Tuladhar NR. Status of multidrug resistance and extended spectrum betalactamase producing strains causing lower respiratory tract and urinary tract infections among patients attending Tribhuvan University Teaching Hospital [abstract]. J NAMLS. 2005; 7:30.

6. Livermore DM, Andrews JM, Hawkey PM, Ho PL, Keness Y, Doi $\mathrm{Y}$, et al. Are susceptibility tests enough, or should laboratories still seek ESBLs and carbapenemases directly? J Antimicrob Chemother. 2012; 67:1569-77. http://dx.doi.org/10.1093/jac/dks08 8

7. Henry D. Isenberg. Clinical 10:867-78.
Microbiology Procedures Handbook. 2nd ed. Washington D.C.: ASM press 2004

8. Clinical and Laboratory Standards Institute. Performance standards for antimicrobial susceptibility testing, 17 th informational supplement. Wayne, PA: CLSI.2007; M100S17.

9. Adams-Haduch JM, Paterson DL, Sidjabat HE, Pasculle AW, Potoski BA, Muto CA, et al. Genetic Basis of Multidrug Resistance in Acinetobacter baumannii Clinical Isolates at a Tertiary Medical Center in Pennsylvania. Antimicrob Agents Chemother. 2008; 52:3837-43. http://dx.doi.org/10.1128/AAC.005 70-08

10. Jacoby GA. Genetics of extended spectrum beta-lactamases. Eur J Clin Microbiol Infect Dis. 1994;

http://dx.doi.org/10.1007/bf02390 679

11. Babypadmini S, Appalaraju B. Extended spectrum-beta lactamases in urinary isolates of Escherichia coli and Klebsiella pneumoniaeprevalence and susceptibility pattern in a tertiary care hospital. Ind J Med Microbiol. 2004; 22:172-4.

12. Coudron PE, Moland ES, Sanders CC. Occurrence and detection of extended-spectrum beta-lactamases in members of the family Enterobacteriaceae at a veterans medical center: seek and you may find. J Clin Microbiol. 1997; 35:2593-7.

13. Ahmed I, Salam A. Extended spectrum beta-lactamases and bacterial resistance. Pak J Med Sci. 2002; 18:151-5.

14. Guthrie R. Community-acquired lower respiratory tract infections. Chest. 2001; 120:2021-34.

http://dx.doi.org/10.1378/chest.120 .6 .2021

15. Chen $\mathrm{CH}$, Huang CC. Molecular epidemiological study of clinical Acinetobacter baumannii isolates: phenotype switching of antibiotic resistance. Ann Clin Microbiol Antimicrob. 2013; 12:21. http://dx.doi.org/10.1186/14760711-12-21

16. Pokhrel BM, Koirala J, Mishra SK, Dahal RK, Khadga PK, Tuladhar NR. Multidrug resistance and extended spectrum beta lactamase producing strains causing lower respiratory tract and urinary tract infection. JIOM. 2006; 28:19-27.

17. Shrestha S, Chaudhari R, Karmacharya S, Kattel HP, Mishra SK, Dahal RK, et al. Prevalence of nosocomial lower respiratory tract infections caused by multidrug resistant pathogens. JIOM. 2011; 33:7-14.

18. Bradford PA. Extended-spectrum beta-lactamases in the 21st century: characterization, epidemiology and detection of this important resistance threat. Clin Microbiol Rev. 2001; 14:933-51.

http://dx.doi.org/10.1128/CMR.14. 4.933-951.2001

19. Gupta E, Mohanty S, Sood S, Dhawan B, Das BK, Kapil A. Emerging resistance to carbapenems in a tertiary care hospital in north India. Ind J Med Res. 2006; 124:95-98.

20. Fritsche T, Sader H, Biedenbach D, Streit J, Jones R. Changing patterns of antimicrobial resistances among bacterial isolates recovered from European patients hospitalised with 
pneumonia: report from the SENTRY Antimicrobial Surveillance Programme (19982004). In Proceedings of the 16th European Congress of Clinical Microbiology and Infectious Diseases: 1-4 April 2006; Nice, France.

21. Paterson DL, Bonomo RA. Extended-Spectrum betaLactamases: a Clinical Update. Clin Microbiol Rev. 2005, 18:657-686. http://dx.doi.org/10.1128/CMR.18. 4.657-686.2005

22. Gur D, Guciz B, Hascelik G, Esel D, Sumerkan B, Over U, et al. Streptococcus pneumoniae penicillin resistance in Turkey. J Chemother. 2001, 13:541-545. http://dx.doi.org/10.1179/joc.2001. 13.5.541

23. Subha A, Ananthan S. Extended spectrum beta lactamase (ESBL) mediated resistance to third generation cephalosporins among Klebsiella pneumoniae in Chennai.

24. Thapa B, Adhikari P, Mahat K, Chhetri MR, Joshi LN. Multidrugresistant nosocomial Citrobacter in a hospital in Kathmandu. Nepal Med Coll J. 2009, 11(3):195-199.
25. Gunseren F, Mamikolu L, Ozturk S, Yucesoy M, Biberoglu K, Yulug $\mathrm{N}$, et al. A surveillance study of antimicrobial resistance of gramnegative bacteria isolated from intensive care units in eight hospitals in Turkey. J Antimicrob Chemother. 1999, 43:373-378. http://dx.doi.org/10.1093/jac/43.3. 373

26. Kucukates E. Antimicrobial resistance among Gram-negative bacteria isolated from intensive care units in a Cardiology Institute in Istanbul, Turkey. Jpn J Infect Dis. 2005, 58:228-231.

27. Oudhuis GJ, Hoogkamp-Korstanje VJAA, Stobberingh EE. Antimicrobial resistance in Escherichia coli and Pseudomonas aeruginosa from Intensive care units in the Netherlands, 1998-2005. Int J Antimicrob Agents. 2008, 31:5863.

http://dx.doi.org/10.1016/j.ijantimi cag.2007.08.009

28. Ho PL, Chow KH, Yuen KY, Ng WS, Chau PY. Comparison of novel, inhibitor-potentiated discdiffusion test with other methods for the detection of extended- spectrum beta-lactamases in Escherichia coli and Klebsiella pneumoniae. J Antimicrob Chemother. 1998, 42:49-54.

http://dx.doi.org/10.1093/jac/42.1. 49

29. Yan JJ, Wu SM, Tsai SH, Wu JJ, Su IJ. Prevalence of SHV-12 among clinical isolates of Klebsiella pneumoniae producing extendedspectrum beta-lactamases and identification of a novel AmpC enzyme (CMY-8) in Southern Taiwan. Antimicrob. Agents Chemother. 2000, 44:1438-1442. http://dx.doi.org/10.1128/AAC.44. 6.1438-1442.2000

30. Steward CD, Rasheed JK, Hubert SK, Biddle JW, Raney PM, Anderson GJ, Williams PP, Brittain KL, Oliver A, McGowan JE Jr, Tenover FC: Characterization of clinical isolates of Klebsiella pneumoniae from 19 laboratories using the National Committee for Clinical Laboratory Standards extended-spectrum beta-lactamase detection methods. J Clin Microbiol. 2001, 39:2864-2872. http://dx.doi.org/10.1128/JCM.39. 8.2864-2872.2001 